PROCEEDINGS OF THE

AMERICAN MATHEMATICAL SOCIETY

Volume 127, Number 5, Pages 1463-1471

S 0002-9939(99)04661-4

Article electronically published on January 29, 1999

\title{
WEIGHTED INTEGRABILITY OF DOUBLE TRIGONOMETRIC SERIES
}

\author{
CHANG-PAO CHEN AND XIN-RONG HUANG
}

(Communicated by Christopher D. Sogge)

\begin{abstract}
We study the double trigonometric series whose coefficients $c_{j k}$ are such that $\sum_{j=-\infty}^{\infty} \sum_{k=-\infty}^{\infty}\left|c_{j k}\right|<\infty$. Then its rectangular partial sums converge uniformly to some $f \in C\left(T^{2}\right)$. We give sufficient conditions for the Lebesgue integrability of $\{f(x, y)-f(x, 0)-f(0, y)+f(0,0)\} \phi(x, y)$, where $\phi(x, y)=1 / x y, 1 / x$, or $1 / y$. For certain cases, they are also necessary conditions. Our results extend those of Boas and Móricz from the one-dimensional to the two-dimensional series.
\end{abstract}

\section{INTRODUCTION}

Let $T^{2} \equiv[-\pi, \pi] \times[-\pi, \pi]$. Denote by $s_{m n}(x, y)$ the rectangular partial sums of the double trigonometric series

$$
\sum_{j=-\infty}^{\infty} \sum_{k=-\infty}^{\infty} c_{j k} e^{i(j x+k y)},
$$

where

$$
\sum_{j=-\infty}^{\infty} \sum_{k=-\infty}^{\infty}\left|c_{j k}\right|<\infty
$$

The Weierstrass M-test theorem implies that $s_{m n}(x, y)$ converges uniformly to some $f \in C\left(T^{2}\right)$ as $\min (m, n) \rightarrow \infty$. In [C1], the first author considered the following two conditions:

$$
\begin{gathered}
c_{j k} \rightarrow 0 \quad \text { as } \quad \max (|j|,|k|) \rightarrow \infty, \\
\sum_{j=-\infty}^{\infty} \sum_{k=-\infty}^{\infty}\left(|j|^{\top}\right)^{\alpha}\left(|k|^{\top}\right)^{\beta}\left|\Delta_{11} c_{j k}\right|<\infty,
\end{gathered}
$$

where $\xi^{\top}=\max (\xi, 1), 0<\alpha, \beta<1$, and

$$
\Delta_{p q} c_{j k}=\sum_{s=0}^{p} \sum_{t=0}^{q}(-1)^{s+t}\left(\begin{array}{l}
p \\
s
\end{array}\right)\left(\begin{array}{l}
q \\
t
\end{array}\right) c_{j+s, k+t} .
$$

Obviously, (1.2) implies (1.3). He proved that

Received by the editors September 1, 1997.

1991 Mathematics Subject Classification. Primary 42B99, 42A16.

This research was supported by National Science Council, Taipei, R.O.C., under Grant \#NSC 86-2115-M-007-012. 
Theorem A (Chen [C1]). Let $0<\alpha, \beta<1$. Assume that conditions (1.3) and (1.4) are satisfied. Then $|x|^{-\alpha}|y|^{-\beta}|f(x, y)| \in L^{1}\left(T^{2}\right)$ and

$$
\iint_{T^{2}}\left|s_{m n}(x, y)-f(x, y)\right|\left(|x|^{-\alpha}|y|^{-\beta}\right) d x d y \rightarrow 0 \quad \text { as } \quad \min (m, n) \rightarrow \infty .
$$

This result extends and generalizes [B3, Theorems $4.1 \& 4.2]$ and [Ma, Theorem 4]. Conditions (1.3), (1.4) imply

$$
\sum_{j=-\infty}^{\infty} \sum_{k=-\infty}^{\infty}\left(|j|^{\top}\right)^{\alpha-1}\left(|k|^{\top}\right)^{\beta-1}\left|c_{j k}\right|<\infty
$$

This is equivalent for the case when $\Delta_{11} c_{j k} \geq 0$ for $-\infty<j, k<\infty$. Obviously, (1.5) reduces to (1.2) for the case $\alpha=\beta=1$. It is excluded in Theorem A. For this case, it is known that $x^{-1} y^{-1} f(x, y)$ may not be Lebesgue integrable on $T^{2}$. Instead of Lebesgue integrability, the improper Riemann integrability of $x^{-1} y^{-1} f(x, y)$, or more generally, $f(x, y) \phi(x, y)$ was examined by the first author in [C2]. His results extend and generalize [Ba], [B1], [M2], [M3]. As for the Lebesgue integrability of $x^{-1} y^{-1} f(x, y)$, several known results have been given by Boas [B2], [B3] and Móricz [M4] for the one-dimensional case, and by Brown-Wang [BW], Móricz [M1], and Papp $[\mathrm{P}]$ for higher dimensions. In [P], Papp proved

Theorem B (Papp $[\mathrm{P}])$. Let (1.1) be a double cosine series. Assume that the following three conditions are satisfied for some $p>1$ :

$$
\begin{gathered}
\sum_{m=1}^{\infty} \sum_{n=1}^{\infty}\left\{\sum_{2^{m-1} \leq j<2^{m}} \sum_{2^{n-1} \leq k<2^{n}}(j k)^{p-1}\left|c_{j k}\right|^{p}\right\}^{1 / p}<\infty, \\
\sum_{m=1}^{\infty} \sum_{n=1}^{\infty} \frac{1}{n}\left\{\sum_{2^{m-1} \leq j<2^{m}} j^{p-1}\left|\sum_{k=n}^{\infty} c_{j k}\right|^{p}\right\}^{1 / p}<\infty \\
\sum_{m=1}^{\infty} \sum_{n=1}^{\infty} \frac{1}{m}\left\{\sum_{2^{n-1} \leq k<2^{n}} k^{p-1}\left|\sum_{j=m}^{\infty} c_{j k}\right|^{p}\right\}^{1 / p}<\infty
\end{gathered}
$$

Then the quotient

$$
\frac{f(x, y)-f(x, 0)-f(0, y)+f(0,0)}{x y} \in L^{1}\left(T^{2}\right)
$$

if and only if

$$
\sum_{m=1}^{\infty} \sum_{n=1}^{\infty} \frac{1}{m n}\left|\sum_{j=m}^{\infty} \sum_{k=n}^{\infty} c_{j k}\right|<\infty .
$$

Papp also derived analogous results for double sine series and double cosine-sine series. His results extend [M4, Theorems 1 \& 2] from the one-dimensional to twodimensional series. In Papp's results, condition (1.6) with $p>1$ is involved. For the limiting case $p=1$, condition (1.6) is transformed into

$$
\sum_{j=-\infty}^{\infty} \sum_{k=-\infty}^{\infty}\left|c_{j k}\right|(\ln |j|)^{\top}(\ln |k|)^{\top}<\infty
$$

The results in this direction for the one-dimensional case were given by Boas [B3] and Móricz [M4]. As for the higher-dimensional case, it is still unknown. The purpose of this paper is to extend Boas's and Móricz's results from the one-dimensional 
to two-dimensional series. We shall prove that condition (1.10) is sufficient to guarantee the validity of (1.9), (see Theorem 2.1). Obviously, the Lebesgue dominated convergence theorem tells us that (1.9) implies the truth of the following assertion:

$$
\lim _{\epsilon, \delta \downarrow 0} \int_{\delta}^{\pi} \int_{\epsilon}^{\pi} \frac{f(x, y)-f(x, 0)-f(0, y)+f(0,0)}{x y} d x d y \text { exists. }
$$

Under certain weaker conditions than (1.10), it will be proved that (1.9), (1.10), and (1.11) are equivalent, (see Corollary 2.6). In this paper, the Lebesgue integrability of $x^{-1}\{f(x, y)-f(x, 0)-f(0, y)+f(0,0)\}$ is also discussed, (see Theorem 2.3 and Corollary 2.8). For details, we refer the reader to the next two sections.

\section{MAIn RESUlts}

We first consider the two-dimensional extension of [M4], that is, the Lebesgue integrability of $x^{-1} y^{-1} f(x, y)$ will be examined.

Theorem 2.1. Let $f$ be the limiting function of series (1.1). If condition (1.10) is satisfied, then $f$ is continuous on $T^{2}$, the assertion (1.9) holds, and

$$
\begin{aligned}
& \iint_{T^{2}}\left|\frac{f(x, y)-f(x, 0)-f(0, y)+f(0,0)}{x y}\right| d x d y \\
& \leq(2 \pi+4)^{2}\left\{\sum_{j=-\infty}^{\infty} \sum_{k=-\infty}^{\infty}\left|c_{j k}\right|(\ln |j|)^{\top}(\ln |k|)^{\top}\right\} .
\end{aligned}
$$

Theorem 2.1 is the two-dimensional extension of [M4, Theorem 4 \& Corollary $3]$. It still holds if we replace (1.10) by (1.3) and (2.2):

$$
\sum_{j=-\infty}^{\infty} \sum_{k=-\infty}^{\infty}\left|\Delta_{11} c_{j k}\right|\left\{\sum_{u=0}^{|j|}(\ln u)^{\top}\right\}\left\{\sum_{v=0}^{|k|}(\ln v)^{\top}\right\}<\infty .
$$

This follows from the Fubini theorem. For double sine series whose coefficients satisfy (1.2), the assertion (1.9) reduces to $x^{-1} y^{-1} f(x, y) \in L^{1}\left(T^{2}\right)$. In this case, the conclusions of Theorem 2.1 can be strengthened in the following way.

Corollary 2.2. Assume that series (1.1) is a double sine series and $f$ is its limiting function. If condition (1.10) is satisfied, then $f$ is continuous on $T^{2}, x^{-1} y^{-1} f(x, y)$ $\in L^{1}\left(T^{2}\right)$, and

$$
\iint_{T^{2}}\left|\frac{s_{m n}(x, y)-f(x, y)}{x y}\right| d x d y=o(1) \quad \text { as } \quad \min (m, n) \rightarrow \infty .
$$

Next, we consider the Lebesgue integrability of $x^{-1} f(x, y)$. In this case, condition (1.10) will be replaced by the following condition:

$$
\sum_{j=-\infty}^{\infty} \sum_{k=-\infty}^{\infty}\left|c_{j k}\right|(\ln |j|)^{\top}<\infty
$$

Theorem 2.3. Let $f$ be the limiting function of series (1.1). If condition (2.3) is satisfied, then $f$ is continuous on $T^{2}$, and

$$
\begin{gathered}
x^{-1}\{f(x, y)-f(x, 0)-f(0, y)+f(0,0)\} \in L^{1}\left(T^{2}\right), \\
x^{-1}\{f(x, y)-f(0, y)\} \in L^{1}\left(T^{2}\right) .
\end{gathered}
$$


Moreover, we have

$$
\begin{aligned}
& \iint_{T^{2}}\left|\frac{f(x, y)-f(x, 0)-f(0, y)+f(0,0)}{x}\right| d x d y \\
& \leq\left(8 \pi^{2}+16 \pi\right)\left\{\sum_{j=-\infty}^{\infty} \sum_{k=-\infty}^{\infty}\left|c_{j k}\right|(\ln |j|)^{\top}\right\}, \\
& \int_{T}\left|\frac{f(x, y)-f(0, y)}{x}\right| d x \leq(2 \pi+4)\left\{\sum_{j=-\infty}^{\infty} \sum_{k=-\infty}^{\infty}\left|c_{j k}\right|(\ln |j|)^{\top}\right\} \quad(y \in T) .
\end{aligned}
$$

Theorem 2.3 remains true if we replace $(2.3)$ by (1.3) and (2.8):

$$
\sum_{j=-\infty}^{\infty} \sum_{k=-\infty}^{\infty}\left|\Delta_{10} c_{j k}\right|\left\{\sum_{u=0}^{|j|}(\ln u)^{\top}\right\}<\infty .
$$

For those double trigonometric series with the property that

$$
c_{-j, k}=-c_{j k} \quad(-\infty<j, k<\infty),
$$

the assertions (2.4) and (2.5) reduce to $x^{-1}\{f(x, y)-f(x, 0)\} \in L^{1}\left(T^{2}\right)$ and $x^{-1} f(x, y) \in L^{1}\left(T^{2}\right)$, respectively. In this case, we have

Corollary 2.4. Assume that conditions (2.3) and (2.9) are satisfied. Then the limiting function $f$ of series (1.1) is continuous on $T^{2}$. Moreover, $x^{-1} f(x, 0) \in$ $L^{1}(T), x^{-1} f(x, y) \in L^{1}\left(T^{2}\right)$, and

$$
\iint_{T^{2}}\left|\frac{s_{m n}(x, y)-f(x, y)}{x}\right| d x d y=o(1) \quad \text { as } \quad \min (m, n) \rightarrow \infty .
$$

Obviously, condition (2.9) is satisfied by the double sine-cosine series, the double sine series, and the series $\sum_{j=1}^{\infty} \sum_{k=-\infty}^{\infty} c_{j k}(\sin j x) e^{i k y}$. Therefore, Corollary 2.4 will apply to these double series.

Finally, we give the two-dimensional extension of [B3, Theorem 5.32]. The next theorem provides us with the converse of Theorem 2.1.

Theorem 2.5. Assume that (1.2) holds and that there exists some positive integer $N_{0}$ such that

$$
\begin{array}{cc}
c_{j k} \geq 0 \quad \text { for } \quad \min (|j|,|k|) \geq N_{0}, \\
\sum_{j=-\infty}^{\infty}\left|c_{j k}\right|(\ln |j|)^{\top}<\infty & \left(|k| \leq N_{0}\right), \\
\sum_{k=-\infty}^{\infty}\left|c_{j k}\right|(\ln |k|)^{\top}<\infty & \left(|j| \leq N_{0}\right) .
\end{array}
$$

Then $(1.9) \Longrightarrow(1.11) \Longrightarrow(1.10)$.

Putting Theorems 2.1 and 2.5 together, we get the following result, which extends [B3, Theorem 5.32] from the one-dimensional to the two-dimensional series.

Corollary 2.6. Under the conditions (1.2) and (2.10)-(2.12), the assertions (1.9), (1.10), and (1.11) are equivalent. 
Obviously, (2.4) implies the truth of the following assertion:

$$
\lim _{\epsilon, \delta \downarrow 0} \int_{\delta}^{\pi} \int_{\epsilon}^{\pi} \frac{f(x, y)-f(x, 0)-f(0, y)+f(0,0)}{x} d x d y \quad \text { exists. }
$$

The following two results give another type of two-dimensional extensions of [B3, Theorem 5.32]. Corollary 2.8 can be derived from Theorems 2.3 and 2.7.

Theorem 2.7. Assume that (1.2) and (2.10) - (2.11) are satisfied by some positive integer $N_{0}$. Then $(2.4) \Longrightarrow(2.13) \Longrightarrow(2.3)$.

Corollary 2.8. Under the conditions (1.2) and (2.10)-(2.11), the assertions (2.3), (2.4), and (2.13) are equivalent.

\section{Proofs of MAin RESUlts}

To derive the main results, the following lemma plays an important role. We leave its proof to the reader.

Lemma 3.1. For $j \neq 0$, we have

$$
2 \ln |j| \leq \int_{T}\left|\frac{e^{i j x}-1}{x}\right| d x \leq(2 \pi+4)(\ln |j|)^{\top},
$$

$$
\ln |j| \leq \int_{0}^{\pi} \frac{1-\cos j x}{x} d x \leq(\pi+2)(\ln |j|)^{\top} .
$$

Proof of Theorem 2.1. The Weierstrass M-test theorem implies that the limiting function $f$ is continuous on $T^{2}$. By (1.10) and Lemma 3.1, we obtain

$$
\begin{aligned}
& \iint_{T^{2}}\left|\frac{f(x, y)-f(x, 0)-f(0, y)+f(0,0)}{x y}\right| d x d y \\
& \leq \sum_{j=-\infty}^{\infty} \sum_{k=-\infty}^{\infty}\left|c_{j k}\right|\left\{\int_{T}\left|\frac{e^{i j x}-1}{x}\right| d x\right\}\left\{\int_{T}\left|\frac{e^{i k y}-1}{y}\right| d y\right\} \\
& \leq(2 \pi+4)^{2}\left\{\sum_{j=-\infty}^{\infty} \sum_{k=-\infty}^{\infty}\left|c_{j k}\right|(\ln |j|)^{\top}(\ln |k|)^{\top}\right\}<\infty .
\end{aligned}
$$

Proof of Corollary 2.2. Let $\tilde{Q}(m, n)$ consist of all $(j, k)$ with $|j|>m$ or $|k|>n$. By (1.10) and Lemma 3.1, we get

$$
\begin{aligned}
& \iint_{T^{2}}\left|\frac{s_{m n}(x, y)-f(x, y)}{x y}\right| d x d y \\
& \quad \leq \sum_{(j, k) \in \tilde{Q}(m, n)}\left|c_{j k}\right|\left\{\int_{T}\left|\frac{e^{i j x}-1}{x}\right| d x\right\}\left\{\int_{T}\left|\frac{e^{i k y}-1}{y}\right| d y\right\} \\
& \quad \leq(2 \pi+4)^{2}\left\{\sum_{(j, k) \in \tilde{Q}(m, n)}\left|c_{j k}\right|(\ln |j|)^{\top}(\ln |k|)^{\top}\right\} \\
& \quad \longrightarrow 0 \quad \text { as } \min (m, n) \rightarrow \infty .
\end{aligned}
$$


Proof of Theorem 2.3. Condition (2.3) ensures the continuity of $f$ on $T^{2}$. By (2.3) and Lemma 3.1, we obtain

$$
\begin{aligned}
& \iint_{T^{2}}\left|\frac{f(x, y)-f(x, 0)-f(0, y)+f(0,0)}{x}\right| d x d y \\
& \leq \sum_{j=-\infty}^{\infty} \sum_{k=-\infty}^{\infty}\left|c_{j k}\right|\left\{\int_{T}\left|\frac{e^{i j x}-1}{x}\right| d x\right\}\left\{\int_{T}\left|e^{i k y}-1\right| d y\right\} \\
& \leq\left(8 \pi^{2}+16 \pi\right)\left\{\sum_{j=-\infty}^{\infty} \sum_{k=-\infty}^{\infty}\left|c_{j k}\right|(\ln |j|)^{\top}\right\}<\infty .
\end{aligned}
$$

This shows (2.4) and (2.6). (2.5) and (2.7) will be proved similarly.

Proof of Corollary 2.4. Let $\tilde{Q}(m, n)$ consist of all $(j, k)$ with $|j|>m$ or $|k|>n$. By (2.3) and Lemma 3.1, we get

$$
\begin{aligned}
\iint_{T^{2}}\left|\frac{s_{m n}(x, y)-f(x, y)}{x}\right| d x d y & \leq 2 \pi\left\{\sum_{(j, k) \in \tilde{Q}(m, n)}\left|c_{j k}\right|\left(\int_{T}\left|\frac{e^{i j x}-1}{x}\right| d x\right)\right\} \\
& \leq\left(4 \pi^{2}+8 \pi\right)\left\{\sum_{(j, k) \in \tilde{Q}(m, n)}\left|c_{j k}\right|(\ln |j|)^{\top}\right\} \\
& \longrightarrow 0 \quad \text { as } \quad \min (m, n) \rightarrow \infty .
\end{aligned}
$$

Proof of Theorem 2.5. It suffices to show (1.11) $\Longrightarrow(1.10)$. By (1.2) and the Weierstrass M-test theorem, we find that

$$
\begin{aligned}
\int_{\delta}^{\pi} \int_{\epsilon}^{\pi} & \frac{f(x, y)-f(x, 0)-f(0, y)+f(0,0)}{x y} d x d y \\
& =\sum_{j=-\infty}^{\infty} \sum_{k=-\infty}^{\infty} c_{j k}\left\{\int_{\epsilon}^{\pi} \frac{e^{i j x}-1}{x} d x\right\}\left\{\int_{\delta}^{\pi} \frac{e^{i k y}-1}{y} d y\right\} \\
& =\left\{\Sigma_{1}(\epsilon, \delta)-\Sigma_{2}(\epsilon, \delta)\right\}+i\left\{\Sigma_{3}(\epsilon, \delta)+\Sigma_{4}(\epsilon, \delta)\right\}
\end{aligned}
$$

where

$$
\begin{aligned}
& \Sigma_{1}(\epsilon, \delta) \equiv \sum_{j=-\infty}^{\infty} \sum_{k=-\infty}^{\infty} c_{j k}\left\{\int_{\epsilon}^{\pi} \frac{\cos j x-1}{x} d x\right\}\left\{\int_{\delta}^{\pi} \frac{\cos k y-1}{y} d y\right\}, \\
& \Sigma_{2}(\epsilon, \delta) \equiv \sum_{j=-\infty}^{\infty} \sum_{k=-\infty}^{\infty} c_{j k}\left\{\int_{\epsilon}^{\pi} \frac{\sin j x}{x} d x\right\}\left\{\int_{\delta}^{\pi} \frac{\sin k y}{y} d y\right\}, \\
& \Sigma_{3}(\epsilon, \delta) \equiv \sum_{j=-\infty}^{\infty} \sum_{k=-\infty}^{\infty} c_{j k}\left\{\int_{\epsilon}^{\pi} \frac{\cos j x-1}{x} d x\right\}\left\{\int_{\delta}^{\pi} \frac{\sin k y}{y} d y\right\}, \\
& \Sigma_{4}(\epsilon, \delta) \equiv \sum_{j=-\infty}^{\infty} \sum_{k=-\infty}^{\infty} c_{j k}\left\{\int_{\epsilon}^{\pi} \frac{\sin j x}{x} d x\right\}\left\{\int_{\delta}^{\pi} \frac{\cos k y-1}{y} d y\right\} .
\end{aligned}
$$

We have assumed (1.11). Therefore, $\lim _{\epsilon, \delta \downarrow 0}\left\{\Sigma_{1}(\epsilon, \delta)-\Sigma_{2}(\epsilon, \delta)\right\}$ exists. Set

$$
g_{j k}(\epsilon, \delta)=\left\{\int_{\epsilon}^{\pi} \frac{\sin j x}{x} d x\right\}\left\{\int_{\delta}^{\pi} \frac{\sin k y}{y} d y\right\} .
$$


Then, for all $j, k$, we have

$$
\lim _{\epsilon, \delta \downarrow 0} g_{j k}(\epsilon, \delta)=\left\{\int_{0}^{\pi} \frac{\sin j x}{x} d x\right\}\left\{\int_{0}^{\pi} \frac{\sin k y}{y} d y\right\} .
$$

Since the integral $\int_{\xi}^{\pi}(\sin n t) / t d t$ is uniformly bounded in $n$ and $0<\xi \leq \pi$, $\left\{g_{j k}(\epsilon, \delta)\right\}_{j, k=-\infty}^{\infty}$ is uniformly bounded on $(0, \pi] \times(0, \pi]$. By (1.2) and the Weierstrass M-test theorem, we find that $\lim _{\epsilon, \delta \downarrow 0} \Sigma_{2}(\epsilon, \delta)$ exists. Thus, $\lim _{\epsilon, \delta \downarrow 0} \Sigma_{1}(\epsilon, \delta)$ exists. Set

$$
P \equiv\left\{(j, k):|j|>N_{0} \text { and }|k|>N_{0}\right\} \quad \text { and } \quad \tilde{P}=\left\{(j, k):|j| \leq N_{0} \text { or }|k| \leq N_{0}\right\} .
$$

Then

$$
\Sigma_{1}(\epsilon, \delta)=\sum_{(j, k) \in P} c_{j k} h_{j k}(\epsilon, \delta)+\sum_{(j, k) \in \tilde{P}} c_{j k} h_{j k}(\epsilon, \delta)=\Sigma_{11}(\epsilon, \delta)+\Sigma_{12}(\epsilon, \delta),
$$

say, where

$$
h_{j k}(\epsilon, \delta)=\left\{\int_{\epsilon}^{\pi} \frac{\cos j x-1}{x} d x\right\}\left\{\int_{\delta}^{\pi} \frac{\cos k y-1}{y} d y\right\} .
$$

For $|j| \leq N_{0}$ and $-\infty<k<\infty$, we have

$$
\begin{aligned}
\left|h_{j k}(\epsilon, \delta)\right| & \leq(\pi+2)^{2}\left(\ln N_{0}\right)^{\top}(\ln |k|)^{\top} \quad(0<\epsilon, \delta \leq \pi), \\
\lim _{\epsilon, \delta \downarrow 0} h_{j k}(\epsilon, \delta) & =\left\{\int_{0}^{\pi} \frac{\cos j x-1}{x} d x\right\}\left\{\int_{0}^{\pi} \frac{\cos k y-1}{y} d y\right\} .
\end{aligned}
$$

Applying (2.12) and the Weierstrass M-test theorem, we conclude that

$$
\lim _{\epsilon, \delta \downarrow 0} \sum_{|j| \leq N_{0}}\left\{\sum_{k=-\infty}^{\infty} c_{j k} h_{j k}(\epsilon, \delta)\right\} \quad \text { exists. }
$$

Similarly, (2.11) implies that

$$
\lim _{\epsilon, \delta \downarrow} \sum_{|k| \leq N_{0}}\left\{\sum_{|j|>N_{0}} c_{j k} h_{j k}(\epsilon, \delta)\right\} \quad \text { exists. }
$$

Therefore, $\lim _{\epsilon, \delta \downarrow 0} \Sigma_{12}(\epsilon, \delta)$ exists and so $\alpha \equiv \lim _{\epsilon, \delta \downarrow 0} \Sigma_{11}(\epsilon, \delta)$ exists. For $(j, k) \in$ $P$, we have $c_{j k} \geq 0$. Hence, Lemma 3.1 leads us to

$$
\begin{aligned}
\infty>\alpha & =\sum_{(j, k) \in P} c_{j k}\left\{\int_{0}^{\pi} \frac{1-\cos j x}{x} d x\right\}\left\{\int_{0}^{\pi} \frac{1-\cos k y}{y} d y\right\} \\
& \geq \sum_{(j, k) \in P}\left|c_{j k}\right|(\ln |j|)^{\top}(\ln |k|)^{\top},
\end{aligned}
$$

and consequently, the desired result follows from (2.11) and (2.12). 
Proof of Theorem 2.7. It can be done by modifying the proof of Theorem 2.5. The essential changes are to replace $(\cos k y-1) / y$ and $\sin k y / y$ by $\cos k y-1$ and $\sin k y$, respectively, for each place where they occur. The other changes are

$$
\begin{aligned}
\int_{\delta}^{\pi} \int_{\epsilon}^{\pi} & \frac{f(x, y)-f(x, 0)-f(0, y)+f(0,0)}{x} d x d y \\
= & \left\{\Sigma_{1}(\epsilon, \delta)-\Sigma_{2}(\epsilon, \delta)\right\}+i\left\{\Sigma_{3}(\epsilon, \delta)+\Sigma_{4}(\epsilon, \delta)\right\}, \\
\left|h_{j k}(\epsilon, \delta)\right| \leq & \left(2 \pi^{2}+4 \pi\right)\left(\ln N_{0}\right)^{\top} \quad\left(|j| \leq N_{0} ;-\infty<k<\infty\right), \\
\left|h_{j k}(\epsilon, \delta)\right| \leq & \left(2 \pi^{2}+4 \pi\right)(\ln |j|)^{\top} \quad\left(-\infty<j<\infty ;|k| \leq N_{0}\right), \\
\infty>\alpha= & \sum_{(j, k) \in P} c_{j k}\left\{\int_{0}^{\pi} \frac{1-\cos j x}{x} d x\right\}\left\{\int_{0}^{\pi}(1-\cos k y) d y\right\} \\
\geq & \pi\left\{\sum_{(j, k) \in P}\left|c_{j k}\right|(\ln |j|)^{\top}\right\} .
\end{aligned}
$$

The condition (1.2) implies

$$
\sum_{|j| \leq N_{0}} \sum_{k=-\infty}^{\infty}\left|c_{j k}\right|(\ln |j|)^{\top} \leq\left(\ln N_{0}\right)^{\top}\left(\sum_{|j| \leq N_{0}} \sum_{k=-\infty}^{\infty}\left|c_{j k}\right|\right)<\infty .
$$

Putting these together with (2.11) yields the desired result.

\section{ACKNOWLEDGMENT}

The authors are grateful to the referee and Professor Jill C. Pipher for valuable suggestions which improved the presentation of this paper.

\section{REFERENCES}

[Ba] N. K. Bary, A Treatise on Trigonometric Series, Pergamon, Oxford, 1964, p. 656.

[B1] R. P. Boas, Integrability of trigonometric series, Duke Math. J. 18 (1951), 787-793. MR 13:549b

[B2] Integrability of trigonometric series III, Quart. J. Math. Oxford Ser. (2) 3 (1952), 217-221. MR 14:867b

[B3] , Integrability Theorems for Trigonometric Transforms, Springer-Verlag, Berlin/ New York, 1967. MR 36:3043

[BW] G. Brown and K. Y. Wang, On a conjecture of F. Móricz, Preprint. CMP 97:13

[C1] C.-P. Chen, Weighted integrability and $L^{1}$-convergence of multiple trigonometric series, Studia Math. 108 (1994), 177-190. MR 95b:42010

[C2] Integrability of multiple trigonometric series and Parseval's formula, J. Math. Anal. Appl. 186 (1994), 182-199. MR 95h:42011

[Ma] M. M. H. Marzuq, Integrability theorem of multiple trigonometric series, J. Math. Anal. Appl. 157 (1991), 337-345. MR 92f:42008

[M1] F. Móricz, Integrability of double lacunary sine series, Proc. Amer. Math. Soc. 110 (1990), 355- 364. MR 90m:42020

[M2] - On the integrability of double cosine and sine series I, J. Math. Anal. Appl. 154 (1991), 452-465. MR 92b:42016

[M3] - Integrability of double cosine-sine series in the sense of improper Riemann integral, J. Math. Anal. Appl. 165 (1992), 419-437. MR 93g:42007

[M4] Integrability theorems for trigonometric series, Acta Sci. Math. (Szeged) 57 (1993), 443-452. MR 95d:42006 
[P] K. F. Papp, Lebesgue integrability theorems for double trigonometric series, Acta Sci. Math. (Szeged) 61 (1995), 331-344. MR 97m:42018

[Z] A. Zygmund, Trigonometric series (2nd ed.), Cambridge Univ. Press, Cambridge, 1968. MR 38:4882

Department of Mathematics, National Tsing Hua University, Hsinchu, Taiwan 30043, Republic of China

E-mail address: cpchen@math.nthu.edu.tw 